\title{
De quelle vie s'agit-il, exactement? Capacité et consentement au Canada
}

U n patient sage parlera avec son médecin de famille de directives préalables, ou directives sur les soins personnels. À l'hôpital, les médecins sages interrogeront leurs patients au sujet de ces directives au moment de l'admission. Malheureusement, cette sagesse ne prévaut habituellement pas. Et comme les lois sur la capacité et le consentement au Canada diffèrent énormément d'une région à l'autre, il en résulte des approches pour le moins incohérentes, comme le démontrent les deux cas qui suivent.

Cas I - en Ontario : Joyce Holland, femme âgée de Toronto atteinte de démence avancée, est inapte à prendre des décisions au sujet de ses soins personnels et a besoin de soins intégraux en établissement de soins de longue durée. Hospitalisée pour une pneumonie par aspiration, elle est admise par la suite à maintes reprises aux soins intensifs. L'équipe des soins intensifs convoque éventuellement une réunion de famille et recommande que $M^{\text {me }}$ Holland ne soit pas réadmise aux soins intensifs mais reçoive plutôt son congé de l'hôpital parce que ses chances de survie sont minces et que les traitements agressifs aux soins intensifs peuvent occasionner de la douleur et des souffrances. Les filles de $M^{\text {me }}$ Holland affirment que ce n'est pas ce que souhaite leur mère, mais elles n'ont aucun document à l'appui. Un médecin des soins intensifs, le $\mathrm{D}^{\mathrm{r}}$ Laura Hawryluck, demande alors à la Commission de révision du consentement et de la capacité de l'Ontario (organisme indépendant qui statue sur des questions relatives à la prise de décisions médicales) de l'autoriser à ne pas administrer à $\mathrm{M}^{\mathrm{me}} \quad$ Holland des traitements essentiels au maintien de la vie. La Commission ordonne aux filles de la patiente de permettre «la non-prestation ou le retrait du soutien par ventilateur ${ }^{1} »$. La famille en appelle de la décision auprès de la Cour supérieure de l'Ontario, laquelle finit par libérer les filles de la patiente de l'ordonnance de la Commission, non parce qu'une approche était plus respectueuse de l'éthique que l'autre, mais parce que le tribunal conclut que la Commission avait mal compris les données médicales. Les filles de $\mathrm{M}^{\mathrm{me}}$ Holland demandent alors que leur mère continue à recevoir des soins médicaux complets, y compris les traitements essentiels au maintien de la vie. Il s'est écoulé environ un an entre la réunion de la famille et la décision du tribunal.

Cas 2 - en Alberta : $M^{\text {me }} \mathrm{T}$, patiente âgée dont l'état ressemble à celui de $M^{\text {me }}$ Holland, est hospitalisée en soins actifs pour une pneumonie par aspiration. Son état se détériore et on demande une consultation aux soins intensifs pour envisager une ventilation mécanique. $M^{\text {me }} \mathrm{T}$ n'a pas de directives préalables décrivant ses désirs ou désignant un fondé de pouvoir. En Alberta, dans les situations d'urgence où le patient est incapable de donner un consentement éclairé et où aucun tuteur n'a été désigné, la pratique consiste à permettre le traitement si deux médecins indépendants évaluent le patient et signent un certificat indiquant que celui-ci a besoin de traitements ou d'examens, mais ne peut y consentir ${ }^{2}$. En moins de 90 minutes, le médecin des soins intensifs, après consultation avec le médecin chargé de l'admission, décide de ne pas admettre $M^{\text {me }} \mathrm{T}$ aux soins intensifs à cause de la gravité sousjacente de son état et du pronostic médiocre. Ils informent par la suite la fille de $\mathrm{M}^{\mathrm{me}} \mathrm{T}$ de leur décision et modifient les soins pour les axer sur le confort. $\mathrm{M}^{\mathrm{me}} \mathrm{T}$ meurt plusieurs heures plus tard.
Ces cas illustrent les extrêmes de l'approche disparate du Canada en matière de lois sur la capacité et le consentement. Il en résulte des approches différentes sur le plan de la prise en charge, et des défis pour les cliniciens, les patients et les familles.

Et que dire des personnes qui changent de province ou de territoire? L'Alberta, par exemple, accueille beaucoup de personnes de l'est du pays. Combien de ces gens savent qu'il faut rédiger une directive personnelle pour désigner un fondé de pouvoir? Une famille qui ne le sait pas peut se voir empêchée de prendre des décisions et obligée de s'adresser aux tribunaux pour obtenir la tutelle. En notre qualité de médecins, il nous incombe de veiller à ce que les patients connaissent les préoccupations particulières à leur province ou territoire en matière de capacité et de consentement.

Les médecins ont un défi difficile à relever lorsque vient le moment de décider des soins appropriés pour leurs patients en fin de vie. Il leur faut sonder les désirs et les attentes du patient, que les membres de sa famille et ses amis sont les mieux placés pour faire connaître. Il ne faut pas invoquer, pour éviter de consulter l'entourage, le fait que l'Alberta n'a pas de mesure législative autorisant expressément les membres de la famille d'un patient à agir en fondés de pouvoir. Toutes les lois devraient au moins reposer sur une norme nationale claire obligeant à inclure les membres de la famille et les soignants des patients dans le processus décisionnel. Les lois sur le consentement servent aussi de repères pour guider les médecins quant au moment où il faut demander le consentement pour dispenser un traitement. Ces repères differrent toutefois lorsqu'il s'agit de savoir si le médecin doit obtenir un consentement pour refuser un traitement non indiqué sur le plan médical ou pour mettre fin à un tel traitement. Au JAMC, nous sommes d'avis qu'un débat national sur ces enjeux s'impose pour que tous les intéressés puissent se faire entendre. Il faut modifier ces approches diamétralement opposées que des lois provinciales mal conçues sur le consentement et la capacité imposent aux Canadiens. Une mesure d'incitation pourrait consister à relier l'implantation de normes nationales aux paiements de transfert fédéraux. La population canadienne mérite des soins d'une qualité équitable.

\section{Sharon Straus MD MSc}

Rédactrice de la section Synthèse, JAMC

Tom Stelfox MD PhD

Professeur adjoint, Département des soins intensifs Université de Calgary, Calgary (Alta.)

Avec l'équipe de rédaction de l'éditorial (Paul C. Hébert MD MHSc, Matthew B. Stanbrook MD PhD, Barbara Sibbald BJ, Ken Flegel MDCM MSc, Noni MacDonald MD MSc et Amir Attaran LLB DPhil)

\section{RÉFÉRENCES}

I. Scardonic. Hawryluck (2004), CanLII 34326 (C.S. ON).

2. Collège des médecins et chirurgiens de l'Alberta. Guidelines : competency assessment and surrogate decision making : responsibilities and roles of a physician. Edmonton : Le Collège; 2002. Disponible à : www.cpsa.ab.ca/publicationsresources/attachments _policies/Competency\%20Assessment $\% 20$ and $\% 20$ Surrogate $\% 20$ Decision $\% 20$ Making.pdf (consulté le I8 octobre 2007). 